\title{
Castillejo de Monteagudo (Murcia, España). Análisis integral para la conservación de estructuras islámicas en regiones sísmicas
}

Castillejo of Monteagudo (Murcia, Spain). Comprehensive analysis for Islamic structures conservation in seismic regions

\section{María José Serrano Latorre a, Adolfo Alonso Durá b, Pedro Enrique Collado Espejo c, Santiago Tormo Esteve ${ }^{d}$}

${ }^{a}$ Patrimonio Inteligente S.L., Murcia, Spain, mariajoseserrano@patrimoniointeligente.com

${ }^{\mathrm{b}}$ Universitat Politècnica de València, Valencia, Spain, aalonsod@mes.upv.es

${ }^{\mathrm{c}}$ Universidad Politécnica de Cartagena, Cartagena, Spain, pedroe.collado@upct.es

${ }^{\mathrm{d}}$ Universitat Politècnica de València, Valencia, Spain, santores@arq.upv.es

\begin{abstract}
The Castillejo of Monteagudo, which was built on second half of the twelfth century, is a fortified palace situated on rural suburbs of Murcia. It is $4 \mathrm{~km}$ to the northeast from the historic center of the city. This monument was part of a big almunia constitued by several emblematic buildings and some important hydraulic and agricultural infrastructures. It is unique in the Iberian Peninsula so its study and conservation are important to understand a not so well known historical period. The walls of this building were constructed in rammed earth and they contained one of the most important cruised patio of hispanic-muslim architecture. In spite of been listed as a Cultural Interest property its conservation state is awful. Its abandonment hinders its preservation for future generations. The Region of Murcia is an area with seismic activity. So, considering the place where the Castillejo is located, a seismic-structural study from a virtual model is executed to check its behavior in front of an earthquake. The results supply relevant information of both its current structural conservation state and its behavior or damages in case of a seismic event. That allow us to assess how urgent an intervention is and also it assures the correct way of conservation, restoration and maintenance.
\end{abstract}

Keywords: Castillejo, Monteagudo, structural-conservation, seismic analysis.

\section{Introducción}

El Castillejo de Monteagudo (Murcia, España), Qasr Ibn Sa’d según fuentes árabes históricas, es un palacio fortificado del siglo XII que forma parte del conjunto monumental de Monteagudo. Se ubica a unos $5 \mathrm{~km}$ de la ciudad de Murcia, en dirección noreste. La complicada orografía que posee, dominando la Vega del Segura, ha favorecido durante siglos el asentamiento de numerosas civilizaciones, desde la prehistoria hasta la actualidad. Su situación estratégica favorecía el control de todo el valle, desde Sierra Espuña hasta Orihuela, además de facilitar la comunicación con otros núcleos de población cercanos y, por supuesto, proporcionaba la defensa que sus habitantes precisaban. Por otro lado, la proximidad del Río Segura y la fertilidad de sus tierras aseguraban el abastecimiento de quienes habitaran este lugar. Todo ello hizo que este enclave no pasara desapercibido para los emires murcianos que establecieron así una de las mayores al- 
munias conocidas y que se conservan en la Península Ibérica.

Si bien ya era conocida la existencia de algunos restos como albercas, acequias, norias y otros elementos propios de una explotación agraria islámica tradicional, los trabajos arqueológicos realizados recientemente en este entorno histórico están documentando un conjunto de construcciones de cierta envergadura que muestran, no solo el poder económico de aquellos que lo construyeron sino también, la importancia social de este conjunto histórico en su momento. Por supuesto, en este lugar no podían faltar grandes edificios y palacios, aunque actualmente solo se conservan restos de tres de ellos: el Castillo de Monteagudo (fortaleza defensiva), el Castillejo de Monteagudo (palacio fortificado) y el Castillo de Larache (residencia menor).

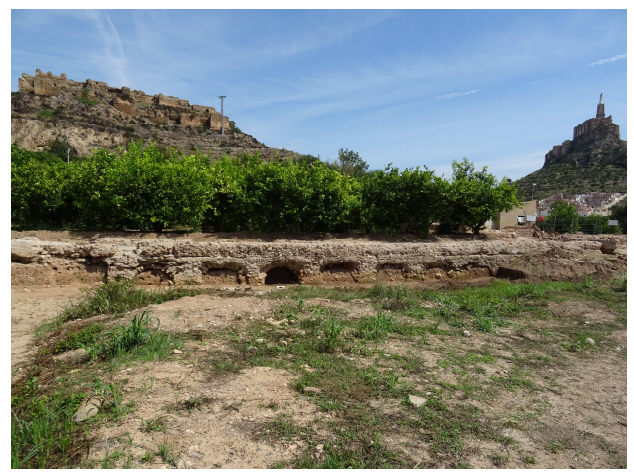

Fig. 1. Vista general del Castillejo de Monteagudo (arriba-izquierda), el Castillo de Monteagudo (arribaderecha) y un acueducto en los alrededores del Castillejo (centro), (Autores).

A pesar de que existen reseñas anteriores que atestiguan la existencia de una fortaleza en Monteagudo, históricamente la construcción del Castillejo y de todo este conjunto se atribuye al emir Ibn Mardanish, conocido como Rey Lobo, gobernador de la ciudad de Mursiya (actual Murcia), entre 1147 y 1172 (Torres, 1934).

El Castillejo de Monteagudo debió cumplir la función de castillo-palacio rural, lugar de recepción diplomática y residencia de recreo del emir. Este edificio albergó un patio de crucero cuya simetría arquitectónica creó un precedente en la arquitectura hispano-musulmana tanto, que para algunos historiadores este patio sirvió de modelo para la construcción del famoso Patio de los Leones de la Alhambra (Granada). No obstante, su reconocimiento como Monumento Histórico Artístico Nacional (Decreto 4 de Junio de 1931), no fue suficiente para asegurar la protección y conservación de este patio. Con posterioridad, este conjunto fue declarado como Bien de Interés Cultural con categoría de Sitio Histórico (Decreto 37/2004), sin embargo, su estado de deterioro y degradación se ha ido dilatando en el tiempo hasta la actualidad.

Recientemente, el Castillejo de Monteagudo ha pasado a ser propiedad del Ayuntamiento de Murcia con lo que se espera, se revierta la situación actual de ruina y abandono que posee y, en un futuro próximo se inicien las necesarias obras de consolidación, restauración y puesta en valor que este monumento demanda.

\section{Objetivos}

La Región de Murcia es una de las áreas con mayor actividad e incidencia sísmica de la Península Ibérica, por lo que resulta de especial interés conocer el estado de conservación de sus estructuras históricas. En estos casos, analizar y determinar el comportamiento de las estructuras frente al sismo sería de gran importancia para valorar la urgencia de intervención tanto, como la necesidad de consolidación, estabilización o conservación estructural para así, en la medida de lo posible, minimizar los daños que pueda provocar un terremoto de cierta envergadura (como en Lorca, 11 de mayo de 2011). Por tanto, los objetivos que persigue el presente estudio son dos, por un lado, el análisis del comportamiento estructural del monumento ante movimientos sísmicos y, por otro, de forma paralela, determinar la necesidad de intervención estructural que precisa el Castillejo para asegurar su correcta conservación.

\section{Cuestiones previas}

Para llevar a cabo este tipo de estudio es necesario realizar un análisis integral de la construcción y de todos los factores externos, que puedan afectar directamente sobre el comportamiento estructural del monumento, como pueden ser las 
características del terreno o las variables del propio seísmo.

Con respecto a la construcción puede ser relevante conocer los antecedentes históricos, el diseño, la tipología y técnicas constructivas que emplearon para su levantamiento, los materiales que componen la estructura portante del bien, o el estado de conservación que posee, tanto como, los agentes de degradación que están afectando al monumento.

Así mismo, son muchos los parámetros externos a la propia construcción que son de especial interés para este tipo de estudios. La naturaleza del terreno o sus características geofísicas y tectónicas podrán ser determinantes para el comportamiento de las estructuras que asientan sobre él, así como la magnitud y la localización del epicentro del terremoto.

\section{Antecedentes constructivos}

Según las fuentes históricas, el Castillejo de Monteagudo se mandó a construir bajo el mandato del emir Ibn Mardanish, gobernador de la ciudad de Murcia durante la segunda mitad del siglo XII. Este personaje, conocido por los cristianos como Rey Lobo, se hizo con el control de Sarq al-Andalus, haciendo de Murcia la segunda capital del territorio hispano-musulmán. Precisamente su elevado poder político y económico le permitieron realizar grandes obras de construcción y reforma, como la renovación de parte de la muralla de la ciudad de Mursiya (actual Murcia) o la gran almunia de recreo que levantó en Monteagudo.

Las obras de nueva planta que se atribuyen a este personaje poseen algunas particularidades que las hacen reconocibles. En todas ellas se advierte el carácter defensivo, pero es la disposición de torres cuadrangulares muy próximas entre sí lo que realmente destaca en sus construcciones, siendo la superficie de sus torres igual o mayor a la de sus lienzos. A esta cuestión, y con especial reseña en el caso del Castillejo, se suma el remate de las esquinas en ángulo entrante (conformado por la unión de dos torres en esquina) y no saliente como es habitual ver en este tipo de arquitectura.

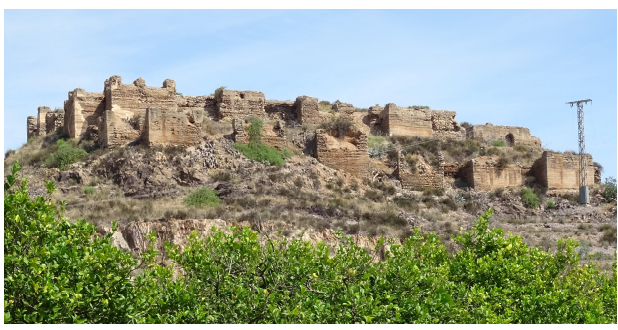

Fig. 2. Lienzos del recinto superior e inferior (alzado suroeste) del Castillejo (Autores).

La construcción del Castillejo se podría dividir en dos cuerpos o dos recintos anexos, siendo el recinto principal o superior donde se generaba el espacio habitable del palacio, y el recinto inferior, ubicado a unos cuatro metros por debajo del superior en su extremo suroeste. De planta rectangular, el recinto superior está formado por cinco torres cuadrangulares salientes en sus lados mayores y tres en sus lados menores, mientras el recinto inferior se compone seis torres en su lado mayor y dos en sendos lados menores. La organización funcional del edificio giraba en torno a un gran patio de crucero cuyos andenes cruzados elevados generaban cuatro parterres o jardines a un nivel inferior, y en sus extremos menores, dos pequeñas albercas abrían paso a sendos salones de recepción. Esta configuración y la época en que se construyó hace que se considere el Castillejo como el primer referente de la simetría arquitectónica palatina andalusí (Torres, 1958).

\subsection{Diseño}

El diseño o la composición formal de un edificio es una de las cuestiones más importantes a tener en cuenta para analizar el comportamiento estructural de una edificación. Como bien se ha comentado, una de las peculiaridades del Castillejo es la disposición de torres muy próximas entre sí y la terminación de sus esquinas en ángulo entrante. Así, la inspección visual realizada in situ puso el foco de atención en estos puntos singulares. Y es que, aunque las esquinas salientes de las torres del Castillejo están ejecutadas como una unidad formal, es decir, sin discontinuidad aparente de ejecución, no sucede lo mismo con las esquinas entrantes, donde se aprecia a simple vista la discontinuidad entre el cuerpo 
de la torre y el muro interior que delimita todo el recinto superior del monumento. Este hecho es apreciable y se repite prácticamente en todas las torres del recinto superior, lo que conduce a pensar que pudiera tratarse de una fase constructiva llevada a cabo durante la ejecución del propio monumento.
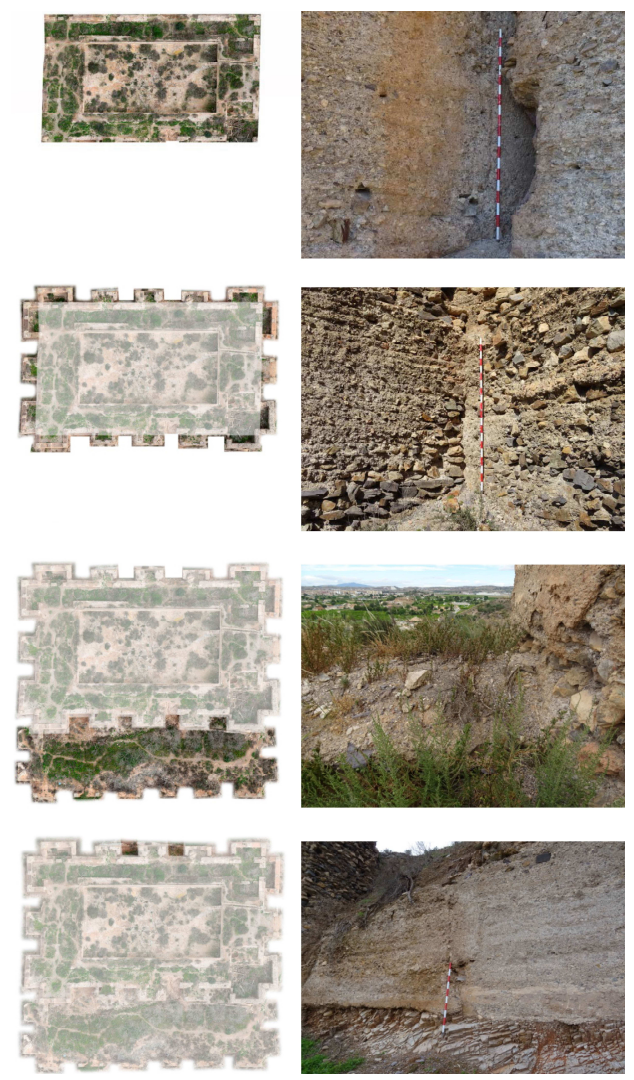

Fig. 3. Fases constructivas detectadas en el Castillejo de Monteagudo (Autores).

De manera semejante sucede lo mismo en otros puntos de este palacio islámico. Por un lado, la construcción y adhesión del recinto inferior, ubicado a cuatro metros por debajo del recinto superior en su lado suroeste, debió proyectarse con posterioridad, o en todo caso, fue adherido al recinto superior tras la construcción de las torres, lo que demuestra una nueva fase constructiva del edificio. Si bien, no se puede obviar que existen ciertas diferencias entre el recinto superior e inferior, no solo por el tamaño y número de sus to- rres, sino también por la composición de las mismas, ya que las del recinto superior fueron concebidas como torres huecas mientras las del recinto inferior se presentan macizas. Y, por último, se pudo detectar el macizado de dos de los lienzos ubicados en su cara noreste, que como sucede en el caso del recinto inferior, debió ejecutarse con posterioridad a las torres, independientemente del tiempo transcurrido entre ambas fases.

Por tanto, como resumen, durante este estudio se pudieron contabilizar hasta cuatro fases constructivas: construcción del recinto rectangular superior, unión de las torres al recinto superior, adhesión del recinto inferior en el lado suroeste, macizado de dos lienzos en su lado noreste.

El análisis metrológico del Castillejo es una de las incógnitas que aún no tienen respuesta. Tras haber realizado una toma de datos de cerca de 200 referencias en todo su perímetro, únicamente se ha podido desprender que son múltiples las medidas empleadas, que se suceden de forma anárquica, por lo que resulta prácticamente imposible unificar y afirmar que fuera solamente una la unidad de medida empleada para levantar sus muros. Si bien es cierto que la mayor parte de estos datos recopilados se encuentran entre los $72 \mathrm{~cm}$ y los $79 \mathrm{~cm}$, por lo que, en base a la ubicación histórico-temporal y la situación política y territorial del momento constructivo del Castillejo, parece factible pensar que se empleara el Codo Mayor Morisco, equivalente a $74 \mathrm{~cm}$ (Serrano, 2018).

\subsection{Materialidad}

Aunque en apariencia se podría decir que las fábricas del Castillejo fueron ejecutadas con la técnica de la tapia hormigonada, el análisis de los materiales empleados, tanto como la técnica constructiva ha propiciado otra teoría.

La localización de mechinales y los restos de agujas hallados en sus muros son la prueba de que la técnica constructiva empleada fuera la tapia. Otra cuestión diferente, a determinar, sería la tipología de tapia que emplearon los alarifes para el levantamiento de estos muros. 
En los muros mejor conservados se aprecian las tongadas de hormigón de cal $(10-15 \mathrm{~cm})$ (Mileto, Vegas, 2014). Sin embargo, en aquellos muros donde la degradación ha sido mayor se observa cómo se ha desprendido esa capa dejando ver en su interior una fábrica compuesta por gran cantidad de mampuestos y considerable tamaño. Por tanto, técnicamente sería imposible que se tratara de una tapia hormigonada, pues la protuberancia de los mampuestos impediría el apisonado de las tongadas de hormigón. Así pues, la hipótesis de la tapia de calicanto o mampuestos parece la más adecuada.

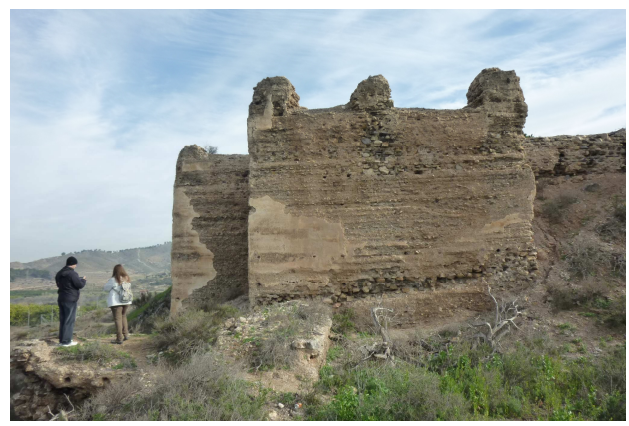

Fig. 4. Torres en esquina noroeste del Castillejo de Monteagudo (Autores).

\subsection{Estado de conservación}

El estado de conservación del monumento es bastante cuestionable. El análisis de las patologías detectadas y el uso de la cámara térmica infrarroja concluyen que el principal factor de degradación que afecta a estas estructuras islámicas es la exposición a los agentes atmosféricos, pero, sobre todo, la falta de conservación y mantenimiento por parte de los anteriores propietarios. Aunque la erosión de las fábricas del Castillejo y la merma de volumen puede parecer insignificante, sobre todo teniendo en cuenta su antigüedad, estas pérdidas de sección pueden presentarse como puntos débiles y comprometer la estabilidad de las fábricas ante un movimiento sísmico de cierta magnitud.

\section{Antecedentes ajenos a la construcción}

Para analizar los efectos de un sismo sobre una construcción histórica hay varios parámetros a considerar. Algunos difícilmente predecibles, como la magnitud, la distancia y la profundidad del epicentro del terremoto, pero otros como la naturaleza del terreno o la vulnerabilidad de la propia construcción (diseño y materialidad), pueden dar una aproximación sobre el comportamiento de la estructura y el grado de afección frente a un movimiento sísmico.

La Región de Murcia está considerada un área de actividad sísmica baja-moderada aunque en varias ocasiones se han alcanzado intensidades muy elevadas, causando daños materiales y personales importantes. Dentro de sus límites territoriales se cuentan hasta seis fallas de primer orden. Concretamente, el Castillejo se ubica en la prolongación de la Falla de Alhama y en el ámbito de fractura de la Falla de Carrasco y, por lo que cualquier seísmo originado a lo largo de estos segmentos tectónicos, podría llegar a afectarle. Además, se ha comprobado que el Castillejo está asentado sobre un estrato rocoso de filitas y calcolitas con un estado de trituración importante, lo que indica una elevada y prolongada exposición sísmica, pero a pesar de ello, en sus muros no se aprecian grietas ni deformaciones significativos, lo que demuestra la solidez constructiva e importante resistencia mecánica de sus fábricas.

Para la zona donde se localiza el Castillejo de Monteagudo, la norma actual (NCSE-02) establece valores de aceleración sísmica entre $0,15 \mathrm{~g}$ y $0,16 \mathrm{~g}$, Tras el terremoto de Lorca (2011) estos valores están siendo revisados, dando valores próximos a los 0,23 g en el último mapa de peligrosidad sísmica publicado por el Instituto Geográfico Nacional. También hay que considerar que el Castillejo comparte la orientación con las fallas próximas por lo que, en caso de seísmo, las estructuras más afectadas serían las que se encuentran orientadas en dirección noroestesuroeste.

\section{Metodología}

Para realizar el análisis sísmico del Castillejo se ha usado Angle, un programa de cálculo estructural desarrollado por la Universidad Politécnica de Valencia que permite, en base a un modelo de elementos sólidos finitos, realizar el cálculo nolineal de una estructura y determinar su compor- 
tamiento frente al sismo. Mediante un procedimiento gradual de carga, el programa evalúa el estado de tensiones frente al peso propio y a la acción sísmica, mostrando los posibles daños estructurales.

El análisis constructivo del Castillejo llevó a determinar que, dado que las torres no se encuentran trabadas con el recinto y estructura principal, se consideró que son elementos independientes y, por tanto, ante un seísmo presentarían comportamientos estructurales individualizados. Este dato permitió simplificar el modelo estructural de estudio al poder considerar la unidad de la torre como elemento representativo del conjunto. No obstante, por estar ubicada en la orientación de mayor incidencia sísmica y por conservar restos pictóricos históricos, para este análisis se decidió tomar como modelo teórico la torre ubicada en la esquina suroriental.

Como se ha comentado, la estructura debe estar configurada como un modelo tridimensional de elementos finitos. Para ello, se procedió a descomponer el cuerpo de la torre en elementos hexaédricos sólidos finitos con características materiales definidas. Así, se generó la malla 3D de la torre formada por un total de 5678 sólidos hexaédricos, 7647 nodos y 317 apoyos.

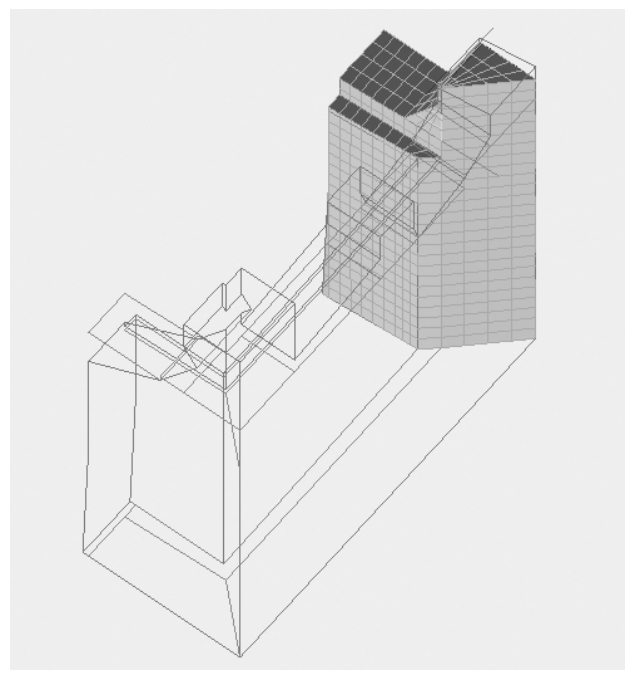

Fig. 5. Detalle de modelización por el método de elementos sólidos finitos (Autores).
Para evaluar el comportamiento estructural de estas fábricas se ha realizado un análisis no lineal con modelo de daño escalar. El proceso de cálculo es incremental de cargas, y para cada instante de cálculo y cada punto se evalúa el índice de daño y su efecto en la pérdida de rigidez $[\sigma=(1-\mathrm{d}) \cdot \mathrm{E} \cdot \varepsilon]$. El estado de cargas se realiza de forma incremental (push-over) en dos fases: en la primera se aplica la carga gravitatoria del peso propio, mientras en la segunda se aplican las acciones sísmicas, evaluando las cargas por el análisis modal espectral (Alonso, Serrano, 2018).

Dado que no se pudieron obtener datos sobre las propiedades materiales de la tapia de calicanto, se plantearon dos supuestos parametrizados: la tapia hormigonada como caso más favorable y la tapia calicostrada como caso más desfavorable.

\begin{tabular}{|l|c|c|}
\hline MATERIAL & $\begin{array}{l}\text { Tapia } \\
\text { hormigonada }\end{array}$ & $\begin{array}{l}\text { Tapia } \\
\text { calicostrada }\end{array}$ \\
\hline $\begin{array}{l}\text { Módulo De- } \\
\text { formación. E, } \\
\text { Mpa. }\end{array}$ & 6000 & 1200 \\
\hline $\begin{array}{l}\text { Coeficiente } \\
\text { Poisson. }\end{array}$ & 0,20 & 0,25 \\
\hline $\begin{array}{l}\text { Resistencia } \\
\text { compresión. } \\
\text { fc, (Mpa). }\end{array}$ & 6,0 & 2,5 \\
\hline $\begin{array}{l}\text { Resistencia } \\
\text { tracción. } \\
\text { ft, (Mpa) }\end{array}$ & 0,70 & 0,05 \\
\hline
\end{tabular}

Tab. 1. Características materiales definidas para el cálculo empleado (Autores).

Asimismo, se tuvo en cuenta que el comportamiento de las estructuras puede variar en función de la dirección y sentido de la onda sísmica, por lo que se consideraron dos nuevos supuestos: empujes en el sentido noreste-suroeste (NE-SO) y empujes en el sentido suroeste-noreste (SO$\mathrm{NE)}$. En definitiva, fueron cuatro los supuestos para realizar el cálculo: tapia hormigonada con empujes en dirección NE-SO (hipótesis 1), tapia calicostrada con empujes en dirección NE-SO (hipótesis 2), tapia hormigonada con empujes en dirección SO-NE (hipótesis 3) y tapia calicostrada con empujes en dirección SO-NE (hipótesis 4).

Mediante la aplicación de un sismo de magnitud moderada con una aceleración sísmica de 0,23 g, 
los gráficos de isovalores resultantes del cálculo demuestran a nivel teórico que, para las cuatro hipótesis planteadas, la estructura de la torre es totalmente estable frente a peso propio (primer gráfico). Sin embargo, frente a las acciones sísmicas (segundo gráfico), aunque los casos de tapia hormigonada muestran un comportamiento más frágil que las de tapia calicostrada, en ninguno de ellos los esfuerzos de tracción superan los valores máximos, de modo que, aunque se podrían producir daños (último gráfico), la torre podría resistir el sismo sin alcanzar la rotura y el colapso estructural.

\section{Conclusiones}

Como cualquier intervención realizada sobre el patrimonio construido, el conocimiento es la base que debe fundamentar cualquier planteamiento. Así pues, para llevar a cabo este estudio ha sido necesario realizar un análisis integral del Castillejo, desde su ubicación históricotemporal, al análisis de los materiales y sistemas constructivos empleados, hasta la valoración de los factores de degradación que están afectando a sus fábricas. Además, para realizar un estudio sísmico estructural resulta imprescindible conocer otros factores externos como pueden ser las características geofísicas y tectónicas del terreno o los antecedentes sísmicos del área donde se ubica, pues podrán ser determinantes para obtener unos resultados lo más próximos a la realidad.

En este caso, el análisis sísmico-estructural realizado ha permitido verificar que las fábricas del Castillejo, frente a un terremoto de magnitud moderada (con una aceleración sísmica de 0,23 g), podrían verse afectadas, aunque sin alcanzar el punto máximo de carga y, por tanto, sus muros serían capaces de resistir el seísmo sin llegar al colapso. Esto se traduce en que el estado estructural de sus muros es bastante bueno y, en consecuencia, no sería necesario realizar una intervención de emergencia para asegurar su estabilidad.

Recientemente ha sido aprobado el Plan Director del Castillo de Monteagudo y, por su lado, el Ayuntamiento de Murcia ha adquirido la propiedad del Castillejo, lo que se considera deberán ser punto de partida para realizar cuantas intervenciones sean necesarias para asegurar la adecuada conservación y puesta en valor de este importante patrimonio cultural.
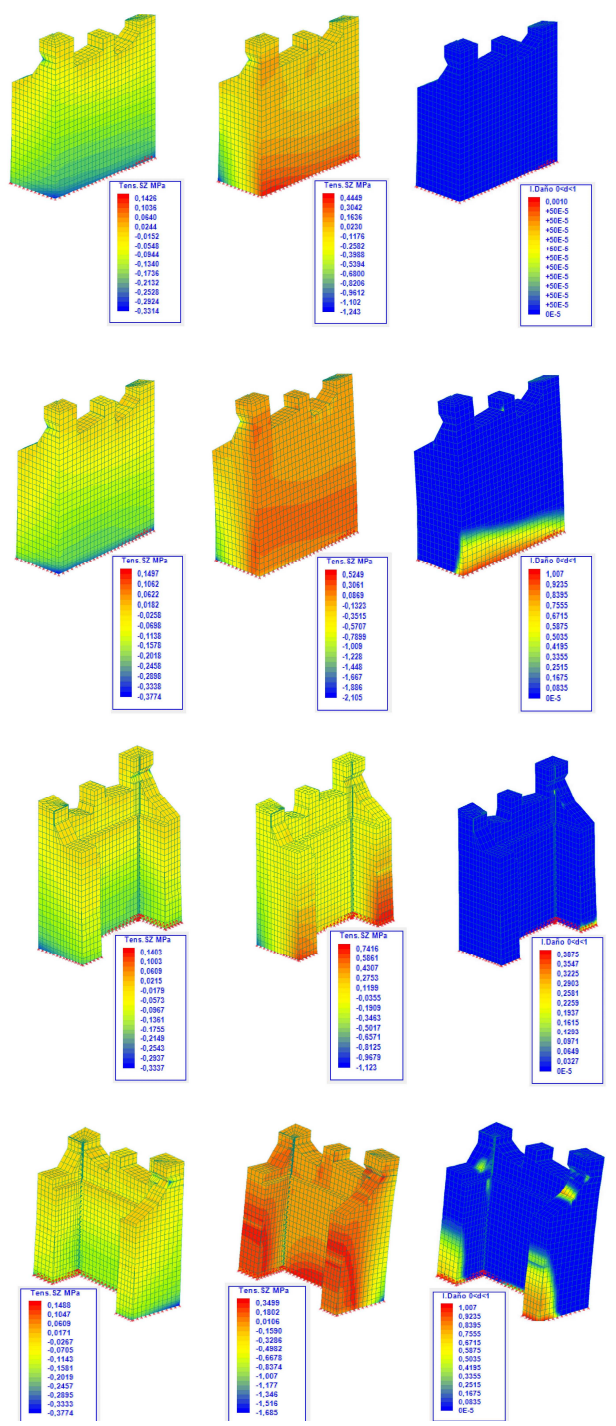

Fig. 6. Gráficos de isovalores según hipótesis planteadas. De arriba a abajo: hipótesis 1 (tapia hormigonada con empujes en dirección NE-SO); hipótesis 2 (tapia calicostrada con empujes en dirección NE-SO); hipótesis 3 (tapia hormigonada con empujes en dirección SO-NE; e hipótesis 4 (tapia calicostrada con empujes en dirección SO-NE), (Autores). 


\section{Bibliography}

Almagro, A. (2008). El concepto de espacio en la arquitectura palatina andalusí, Consejo Superior de Investigaciones Científicas, Madrid.

Alonso, A.; Gomis, J.; Moreno, J.; Llopis, V. (2011). “Arquitectura religiosa: análisis constructivo y estructural”, in Actas del Séptimo Congreso Nacional de Historia de la Construcción, Instituto Juan de Herrera, Madrid, pp. 5564.

Alonso, A.; Serrano, M.J. (2018). "Cálculo sísmico-estructural para el Patrimonio Construido y su aplicación al Castillejo de Monteagudo", in XXIV Jornadas de Patrimonio Cultural de la Región de Murcia, Tres Fronteras Ediciones, Murcia, pp. 91-98.

Collado, P.E.; Serrano, M.J. (2017). "Sustainable proposal for the conservation of Castillejo of Monteagudo (Murcia, Spain)", in Vernacular and Earthen Architecture: Conservation and Sustainability, Taylor \& Francis Group, London, pp. 563-568.

Eiroa, J.A. (2016). "El legado de Ibn Mardanis", in Debates de Arqueología Medieval, n6, Universidad de Granada, pp. 247-258.

Gallego, R.; Arto, I. (2015). "Evaluation of seismic behaviour of rammed earth strutures", in Earthen Architecture: Past, Present and Future, Taylor \& Francis Group, London, pp. 151-156.

Llopis Pulido, V. (2014). La catedral de Valencia: construcción y estructura. Análisis del Cimborrio, Tesis Doctoral (inédita), Universidad Politécnica de Valencia, Valencia.

Mileto, C.; Vegas, F. (2014). La restauración de la tapia en la Península Ibérica. Criterios, técnicas, resultados y perspectivas, TC Cuadernos, Valencia.

Mileto, C.; Vegas, F., coord. (2017). Proyecto COREMANS. Criterios de intervención en la arquitectura de tierra, Ministerio de Educación, Cultura y Deporte, Madrid.

Navarro, J.; Jiménez, P. (1993). “Aproximación al estudio del Castillejo de Monteagudo y otros monumentos de su entorno", in Memorias de Arqueología nº, Consejería de Educación y Cultura, Murcia, pp. 433-453.

Navarro, J.; Jiménez, P. (1995). "El Castillejo de Monteagudo: Qasr Ibn Sa'd”, in Casas y Palacios de Al-Ándalus, Editorial Lunwerg, Barcelona, pp. 63-103.

Navarro, J.; Jiménez, P. (2012). "La arquitectura de Ibn Mardanish; revisión y nuevas aportaciones”, in Aljafería y el arte del Islam occidental en el siglo XI, CSIC, Zaragoza, pp. 291-350.

Serrano, M.J. (2018). "Estudio constructivo y de conservación de estructuras murarias islámicas en zonas sísmicas. El caso de Qasr Ibn Sa'd - Castillejo de Monteagudo", in XXIV Jornadas de Patrimonio Cultural de la Región de Murcia, Tres Fronteras Ediciones, Murcia, pp. 35-42.

Torres, L. (1934). "Monteagudo y el Castillejo en la Vega de Murcia”, Crónica Arqueológica de la España Musulmana. Al-Ándalus, II, pp. 366-372.

Torres, L. (1958). "Patios de crucero”, Crónica Arqueológica de la España Musulmana. Al-Ándalus, XIII, 1, pp. 171191. 